\title{
Role of PET and PET/CT in Anticancer Drug Therapy Response Evaluation
}

\author{
Rakesh Kumar*, $^{*}$, Varun Shandal ${ }^{1}$, Suman Jana ${ }^{2}$, Shamim A. Shamim ${ }^{1}$ and Arun Malhotra ${ }^{1}$ \\ ${ }^{I}$ Department of Nuclear Medicine, All India Institute of Medical Sciences, New Delhi, India \\ ${ }^{2}$ Division of Cardiovascular Medicine, University of Kentucky, Lexington, KY 40536-0200, USA
}

\begin{abstract}
Anti-cancer drug development is a major area of research. New imaging techniques provide a novel method for anti-cancer drug development and monitoring response to therapy by targeting functional metabolic activity at cellular level. The current assessment of the response to therapy in solid tumors based on measurement of the changes in tumor size have many limitations. Positron emission tomography (PET) has been used to measure changes in drug induced metabolism, cellular proliferation and tissue perfusion. Also, changes induced by immuno-modulating drugs such as apoptosis, telomere activity, growth factor levels and many more can be studied using specific radiolabelled PET tracers. Initially PET was used alone without any computed tomography (CT) or magnetic resonance imaging (MRI) hybridization. Since there are few limitations associated with PET alone, a novel combined PET/CT system has recently been built that improves the ability to correctly localize and interpret radiotracer uptake. Hybrid PET/CT scanners provide both the anatomical and functional aspects of the tissue. PET and PET/CT have been found to be very useful in various cancers. In the present study we have investigated role of PET/CT as a predictor of early response to chemotherapy in locally advanced breast cancer patients, lymphoma, pediatric cancer, lung cancer, etc. We have performed more than $14000 \mathrm{PET} / \mathrm{CT}$ at our institute. We have found that fluorine-18 fluoro-deoxy-glucose $\left({ }^{18} \mathrm{~F}\right.$-FDG) PET/CT plays important role in early assessment of treatment response in various cancer patients. A positive PET/CT scans after the completion of therapy is a strong predictor of residual disease, whereas, a negative study is associated with complete remission in these patients.
\end{abstract}

Keywords: FDG PET/CT, anticancer drug, treatment response evaluation.

\section{INTRODUCTION}

There has been significant development in treating the patients with various cancers either through chemotherapy or radiotherapy. Monitoring the therapy response is important in these cases, keeping in mind the long list of severe adverse effects resulting from this therapy. A novel method for monitoring the response of this therapy has been provided by new imaging techniques, more importantly, the functional imaging techniques like PET and PET/CT. For past many years, ${ }^{18}$ F-FDG PET/CT has been extensively used in assessment of chemotherapy treatment response in patients with various cancers. A reduction in FDG uptake in tumor within days to weeks after starting the treatment correlates well with the treatment response and also predicts survival in some cases [1]. Several studies have been conducted and the utility of PET/CT in monitoring the therapy response has been proven, but still, the authenticity of these studies is questionable due to their small sample sizes. Large multicentral clinical trials are needed to authenticate these results. Despite being the fact that PET and PET/CT is very useful for assessing the cytoreductive/ cytotoxic treatment response, it is not a standard choice of investigation for most tumor types yet and definitely, more work is required to make it a standard of care.

*Address correspondence to this author at the Department of Nuclear Medicine, All India Institute of Medical Sciences, New Delhi, India; Tel: 91-11-26588700; Fax: 91-11-26588663; E-mail: rkphulia@yahoo.com
Many chemotherapeutic agents like Trastuzumab, Sunitinib, Imatinib, Lapatinib etc., which are used in various cancer conditions, are cytostatic in nature and halt the tumor growth but do not cause tumor cell death [2-6]. Monitoring the response to these agents using conventional imaging modalities such as CT, MRI would not be helpful, as the basis for assessing the therapy response by these modalities is reduction in size of the tumor. Tumor shrinkage and its dissolution is a complex process which takes significant time to occur, usually weeks to months. It causes loss of precious time while evaluating treatment response using conventional imaging. These modalities also can not differentiate the residual disease from post- therapy changes such as fibrosis and scarring. The newer functional imaging such as PET in association with $\mathrm{CT}$ or MRI is the best imaging available to date. PET scan provides the functional/metabolic status of the tumor and CT/MRI provides the anatomical localization helping the image interpretation with maximum accuracy. This functional information is very helpful to identify early response to therapy so that therapies which are ineffective can be stopped to reduce the expenses, time wastage and its side effects.

\section{BREAST CANCER}

Breast cancer is the most common diagnosed cancer in females and is the second most common cause of cancer deaths after lung and bronchial cancer in this group worldwide. There are multiple options available for the systemic therapy in breast cancer and it is one of the solid 
tumor which is much responsive to the therapy [7]. Treatment options for breast cancer are surgery, chemotherapy and radiotherapy. Various imaging modalities are used for staging, restaging and response evaluation in breast cancer. Conventional techniques available for breast cancer imaging are radiological examinations, such as mammography, Doppler ultrasonography, CT, MRI, magnetic resonance spectroscopy and optical imaging. MRI combined with contrast-enhanced MRI as the functional imaging modality has a diagnostic accuracy of $93 \%$ for identifying tumors showing a pathologic complete response [8].

${ }^{18} \mathrm{~F}$ FDG-PET has been proven to be an accurate imaging modality for staging, restaging recurrent/metastatic disease and for evaluation of therapy response. As it has ability to detect early changes in tumor glucose use, it has been studied worldwide as a method for assessing tumor response to neoadjuvant chemotherapy and it has shown close correlation of changes in ${ }^{18}$ F-FDG uptake with the effectiveness of treatment [9, 10]. PET scanning is usually done at different intervals during and after chemotherapy, but there is no general consensus regarding this. FDG-PET done after the first cycle of chemotherapy for evaluating the therapy response has sensitivity and specificity ranging from $39-100 \%$ and $74-100 \%$, respectively, shown by several studies [9, 11-13]. Sensitivities and specificities after the second cycle were $69-93 \%$ and $75-94 \%$, respectively [1214]. The above data suggests lower accuracy of PET in treatment monitoring after first cycle of chemotherapy. Applying PET after 3 or more cycles of chemotherapy is too late to make effective changes in chemotherapy regime and by this time patient would have already been exposed to ineffective and toxic chemotherapy. Rousseau et al. suggested that treatment response evaluation can be best done after second cycle of chemotherapy [13]. For analysis and assessment of tumor response to therapy, the maximum standard uptake value (SUVmax) method is most widely used. Higher SUV correlates to higher tumor activity and it helps in differentiating responders from non-responders after the chemotherapy. PET is also very useful in evaluating the metastatic disease by scanning whole body in one procedure.

\section{NON SMALL CELL LUNG CANCER}

Lung cancer is the second commonest malignancy in both sexes and is a major cause of cancer related deaths. Non small cell lung cancer (NSCLC) is the most common type of lung cancer. As lung cancer is presented at advanced stages, long term survival rate is low. Since the implementation of PET as a functional imaging modality, early detection of NSCLC is possible, which when used along with CT as PET/CT, helps in detecting nodal and distant metastatic disease more accurately [15]. Detection of widespread metastatic disease early by PET is of great help, as it may prevent the major invasive surgery such as thoracotomy and the patient can be simply put on chemoradiation therapy. Treatment response evaluation using conventional imaging might not be accurate because there is high variability in changing of tumor dimensions after therapy as there are usually different proportions of malignant cells, stroma and inflammatory cells in tumor tissue. Also after the cure of NSCLC, fibrotic tissue may remain which may present as false positive in CT or MRI.
PET in combination with CT or MRI assesses the tumor activity with its proper localization. A baseline scan should always be performed before commencement of the chemotherapy. Post-chemotherapy scan is then compared with the baseline scan for proper interpretation of the treatment response. Disease process is assigned complete response, partial response, stable disease and progressive disease according to PET response criteria in solid tumors (PERCIST) [16]. Some studies have evaluated the role of ${ }^{18}$ F-FDG PET in suspected residual or recurrent NSCLC have shown higher accuracy and ability of providing prognosis than with conventional imaging modalities [1719]. As described in breast cancer section, SUVmax is taken as basis for determining the tumor metabolic status. Some of the histologic types of NSCLC have an intrinsically low SUVmax which may pose a problem in identifying or correlating the grade of tumor with FDG uptake by the tumor. Goudarzi et al. studied 53 patients who had 57 pathologically proven lesions and showed that in 26 lesions having pure bronchioloalveolar carcinoma (BAC) had a median SUVmax of only 1.48 (range, 0.63-4.54) [20]. Out of these BAC lesions, $81 \%$ had SUVmax of less than 2.5, which is a cutoff value usually used to differentiate benign from malignant lesions [20]. PET study results also prognosticate the survival. Nahmias et al. studied ${ }^{18}$ F-FDG PET in 16 patients having NSCLC and evaluated serial changes in the SUV during chemotherapy and subsequently demonstrated that patients having reduction in the SUVmax more than or equal to $50 \%$ between studies performed after 1 and 3 weeks of chemotherapy survived for more than 6 months, whereas patients with SUVmax reduction less than $50 \%$ died within 6 months [21].

\section{GASTROINTESTINAL CANCERS}

There are many cancerous conditions involving gastrointestinal (GI) tract. Esophageal and colorectal cancers are important among all GI cancers in view of their higher incidence and associated mortality with them [22]. Squamous cell carcinoma (SCC) occurs in upper two-third part of esophagus and adenocarcinoma occurs in lower onethird part. SCC is more prevalent than adenocarcinoma of esophagus worldwide, whereas, these both types are equally prevalent in USA.

PET/CT is used in esophageal cancer for pre-therapeutic staging, restaging and for assessing response to therapy. Pretherapeutic staging is important in esophageal cancer to differentiate patients with loco-regional disease from patients having metastatic disease as the treatment regime for each is different than the other. FDG-PET has a sensitivity and specificity of $67 \%$ and $97 \%$ respectively, in metastatic staging of esophageal cancer [23]. To know about clinical consequences after the chemotherapy is sometimes necessary and so, therapy response monitoring becomes important. FDG PET/CT is best available technique for monitoring the therapy response. Treatment response can be assessed either during the course of chemotherapy or after the completion of the therapy. Many studies have demonstrated the sensitivity and specificity of PET/CT in monitoring the treatment response in esophageal cancer ranging from $62-100 \%$ and $55-88 \%$, respectively [24-27].

Recently, because of earlier detection of disease and advancement in chemotherapeutic drugs, the prognosis for 
colorectal cancer patients has improved to a large extent. PET/CT has already proved its role in staging of colorectal cancer and now it is also being used for assessing chemotherapy response. As in other solid tumors, therapy response was being done through conventional imaging in earlier times using response evaluation criteria in solid tumors (RECIST) criteria. According to RECIST criteria, therapy response will be considered only if there is decrease in $30 \%$ diametric dimension of tumor [28]. It has limited value in differentiating residual fibrotic mass or postoperative changes from recurrent disease. Here, PET or PET/CT has overcome this limitation by providing the functional metabolic status of the tumor. Many authors have provided the data regarding the efficacy of PET or PET/CT in monitoring the therapy response in locoregional and metastatic disease in colorectal cancer. Findlay et al. evaluated PET in 18 patients for response evaluation to therapy and found the sensitivity $100 \%$ and specificity $75 \%$ [29]. Dimitrakopoulou-Strauss et al. performed serial 18FFDG PET scans during the chemotherapy course and found a positive correlation between the PET findings and survival times of the patients [30]. de Geus-Oei et al. found increase in mortality rate and disease progression with PET showing worst response after the therapy [31]. Some authors monitored the response after local ablative therapy for treatment of liver metastasis in colorectal cancer and found the positive predictive value and negative predictive value ranging from $80-100 \%$ and $96-100 \%$, respectively [32-36].

\section{HEAD AND NECK CANCERS}

Head and neck cancer is the sixth most common cancer all over the world. Head and neck squamous cell carcinoma (HNSCC) comprises $90 \%$ of these malignancies in western world. Prognosis is worse in non-surgical candidates having advanced disease with less than $10 \%$ five year survival [22]. After the chemotherapy, CT, MRI and FDG PET are considered standard investigations for treatment monitoring in HNSCC.

Some studies have evaluated the efficacy of PET or PET/CT in therapy response monitoring in HNSCC [37-40]. Some of them evaluated PET after induction chemotherapy and others evaluated after complete definitive therapy. In these studies, time of performing PET scans after the completion of chemotherapy varied from 4 weeks to 1 year. Some authors have suggested that the PET study should not be done before 2-3 months after the completion of therapy, as by that time inflammatory changes due to chemotherapy did not subside. So, this reduces the false-positive findings. Some of these studies have also depicted that when PET was done within 1-2 months period after chemotherapy completion, a higher false-negative rate was observed. Reason was formulated that by this early time the smallvolume residual disease usually did not get detected by PET. All these authors have evaluated the sensitivity, specificity, PPV and NPV of PET in therapy response assessment in HNSCC ranging from 40-87\%, 25-91\%, $18-70 \%$ and 50$97 \%$, respectively. After chemotherapy/radiation therapy, focal and asymmetric FDG uptake is generally considered to be residual disease, whereas, non-focal and diffuse FDG uptake is more suggestive of post-radiation inflammation. There is diffuse increased uptake in laryngeal or oropharyngeal areas after the chemoradiotherapy which remains for longer periods. One has to be careful while interpreting the scans in these cases as focal uptake of higher intensity in between the diffuse uptake may be because of ulceration or persistent disease. Post-therapy PET scan also changes the management plan by showing the presence/absence of nodal disease. Neck dissection is the usual procedure followed for the nodal metastatic disease in neck. Ong et al. found that PET/CT findings after therapy reduced the number of planned neck dissections by $75 \%$ [37].

\section{LYMPHOMA}

There are two major types of lymphomas i.e. Hodgkin's disease (HD) and Non-Hodgkin's lymphoma (NHL). There are many different types of NHL, which can be divided into aggressive [fast-growing] and indolent (slow-growing) types and can be classified as either B-cell or T-cell NHL. HD is marked by the presence of a type of cell called as ReedSternberg cell. The two major types of Hodgkin's lymphoma are classical Hodgkin lymphoma and nodular lymphocytepredominant Hodgkin lymphoma. ${ }^{18}$ F-FDG PET/CT has potential value in monitoring the response to treatment in patients with both NHL and HD. PET/CT provides functional and anatomical details in same setting. Treatment response evaluation using $\mathrm{PET} / \mathrm{CT}$ can be done during treatment and evaluation of treatment response after completion of treatment.

Kumar et al. analyzed 19 gastrointestinal lymphoma patients. Of the 19 post-treatment PET scans, 13 showed no pathologic F18-FDG uptake, whereas 6 showed persistent F18-FDG uptake [41]. Among the 13 patients who had negative PET scans, only 1 patient $(7.7 \%)$ relapsed, whereas all 6 patients $(100 \%)$ who had persistent abnormal F18-FDG uptake on posttherapy PET scans relapsed. We concluded that ${ }^{18}$ F-FDG PET has potential value in monitoring the response to treatment in patients with GI tract lymphomas, particularly when pretreatment PET results are positive. In another study, we included 52 patients, of which 19 were of HD and 33 were of NHL. In our study, all patients were of high-grade lymphoma. All patients underwent pre-treatment and post-treatment PET/CT scans on the same dedicated PET/CT scanner. In HD group, SUVmax was 55.5 with a range 4.7-55.5. Post treatment PET/CT scans demonstrated complete resolution of baseline abnormal FDG uptake in 16 of 19 in this group. The sensitivity, specificity, and accuracy of post therapy PET/CT scan in HD group were $67 \%, 94 \%$, and $90 \%$, respectively. In NHL group, post treatment PET/CT scans demonstrated complete resolution of baseline abnormal FDG uptake in 27 of 33 NHL group, Fig. (1). Six patients showed positive PET/CT. The sensitivity, specificity, and accuracy of post therapy PET/CT scan in NHL group were $71 \%, 96 \%$, and $91 \%$, respectively.

Many of the studies showed ${ }^{18}$ F-FDG-PET is a powerful tool for the imaging of aggressive lymphoma. Their results indicate that FDG-PET has reasonable sensitivity and high specificity for evaluation of post-therapy in HD and in NHL [42-46]. These studies showed a sensitivity ranging 70-100 $\%$ and specificity ranging $78-100 \%$. Only few studies were done on PET/CT for evaluation of treatment response in lymphoma. Zhao $\mathrm{J}$ et al. assessed the value of hybrid PET/CT with ${ }^{18}$ F-FDG after 3-4 cycles of chemotherapy for 

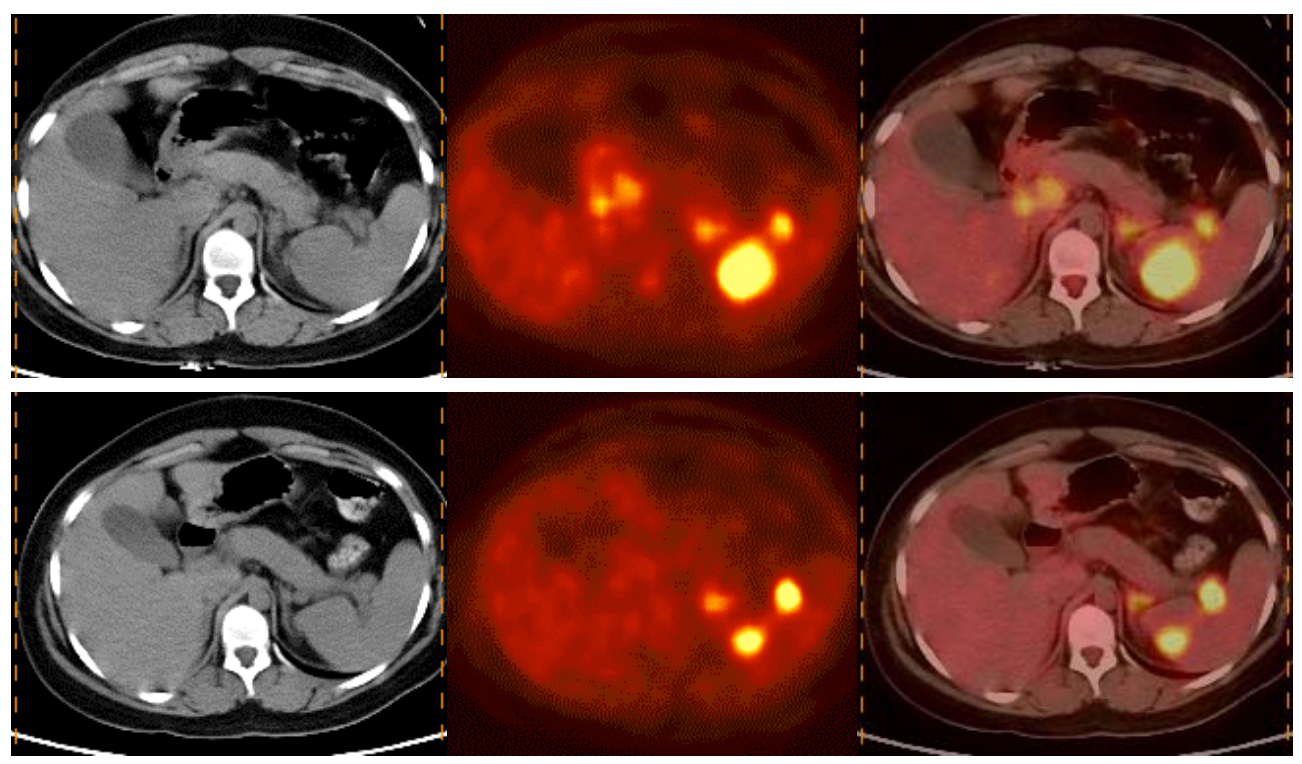

Fig. (1). Pretherapy (upper row) and post therapy (lower row) CT, PET and PET/CT scan showing significant response.

early evaluation of response to therapy and prediction of progression-free survival (PFS) in NHL [47]. Sixty-one consecutive NHL patients were included. After 3-4 cycles of chemotherapy, positive ${ }^{18}$ F-FDG lesions were found in 28 patients, minimal residual uptake (MRU) in 8 and negative scans in 25 patients. In FDG-positive group, 22 patients showed progress and three died. Nine ${ }^{18}$ F-FDG-negative patients and 4 patients from the MRU group relapsed. They concluded that early interim FDG imaging is an excellent and independent predictor of PFS in NHL. An early assessment of chemotherapy response with FDG scans may provide useful information for selection of patients for alternative therapeutic strategies.

\section{GYNAECOLOGICAL MALIGNANCIES}

Gynecological malignancies are major disease burden in women population worldwide. Cervical, endometrial and ovarian cancers together constitute majority of gynecological malignancies. PET and PET/CT can play an important role in evaluating treatment response in these patients. Since FDG is excreted through the urinary tract and also physiologically accumulated in the bowel, this can interfere with the optimal evaluation of abdomen and pelvis using PET/CT. There were several attempts to avoid urinary bladder activity. In our opinion the best method to deal with this problem to give plenty of fluids with diuretics (furosemide) and empty bladder frequently and the hold the urine till bladder is full with non-radioactive urine [this provide negative contrast].

Invasive cancer of the cervix is the second most common genital malignancy in women, worldwide. Surgery is treatment of choice for early cervical cancer. While, locally advanced cervical cancer is treated with definitive radiation therapy with the concurrent administration of intravenous cisplatin chemotherapy. 18F-FDG PET has been used to assess response after chemoradiation for carcinoma of the cervix. The investigators at Washington University in St. Louis, USA found that post treatment metabolic response is predictive of progression free survival after chemoradiation for cervical cancer [48-51]. In a study by Schwarz et al. 92 patients were imaged with 18F-FDG PET after the 3 months of completion of chemoradiation for cervical cancer. The authors demonstrated 3-years PFS rates of $78 \%$ in complete response group, $33 \%$ in partial response group and $0 \%$ in patients who showed progressive disease. A multivariate analysis, only posttherapy metabolic response and pretreatment lymph node status (as defined by 18F-FDG PET) predicted PFS. There are only few studies which evaluate treatment response during the course of radiation therapy for cervical cancer [51,52].

Ovarian cancer is the second most common genital malignancy after uterine cancer in women and has the highest mortality rate among gynecological malignancies in United States and many countries of the world. Avril et al. [53] demonstrated a significant correlation between changes in tumor tracer uptake after the first and third cycles of chemotherapy in ovarian cancer. A higher rate of complete tumor resections was achieved in metabolic responders than in nonresponders, Fig. (2). In addition, metabolic responders had a longer median overall. In another study, Nishiyama et al. [54] concluded that initial SUV derived by FDG PET and percentage change in SUV have the potential to predict response to chemotherapy or chemoradiotherapy in patients with advanced gynecologic cancer. There were 10 responders and 11 nonresponders based on histopathologic analysis. SUV after therapy in responders was significantly lower than that in nonresponders $(p, 0.005)$. When an arbitrary SUV of 3.8 was taken as the cutoff for differentiating between responders and nonresponders after therapy, 18F-FDG PET showed a sensitivity of $90 \%$, a specificity of $63.6 \%$, and an accuracy of $76.2 \%$. Sensitivity of $90 \%$, specificity of $81.8 \%$, and an accuracy of $85.7 \%$ was achieved when an arbitrary percentage change of $65 \%$ is taken as the cutoff for differentiating between responders and nonresponders.

\section{MULTIPLE MYELOMA}

Multiple myeloma constitutes approximately $10 \%$ of all hematologic cancers [54]. It is caused by neoplastic proliferation of plasma cells which presents as bone marrow 
$\mathrm{CA} 125=50 \mathrm{U} / \mathrm{L}$

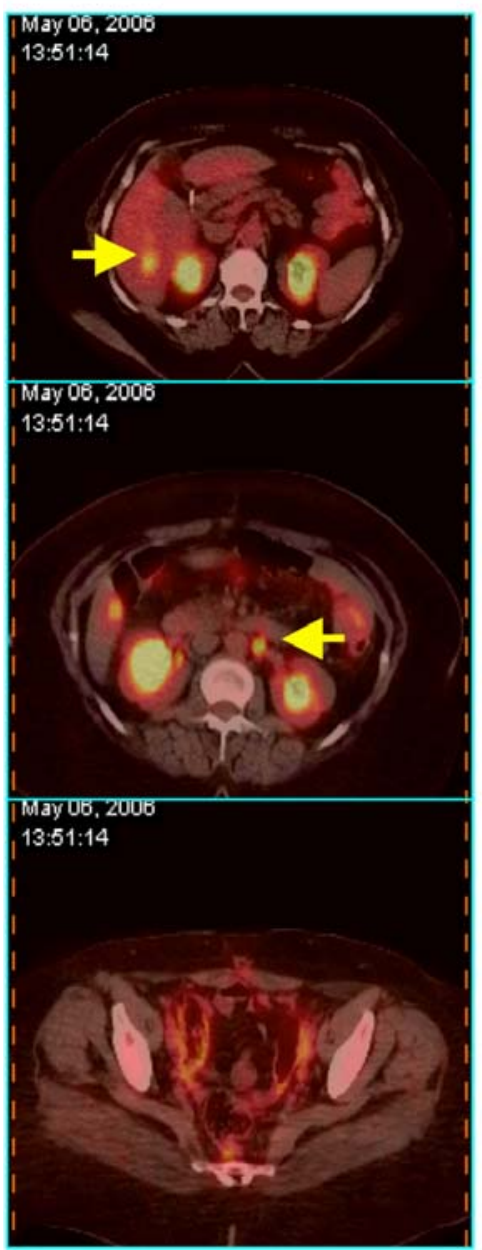

CA125 $=5 \mathrm{U} / \mathrm{L}(6$ cycles of chemo) $\quad$ CA125 $=131 \mathrm{U} / \mathrm{L}$
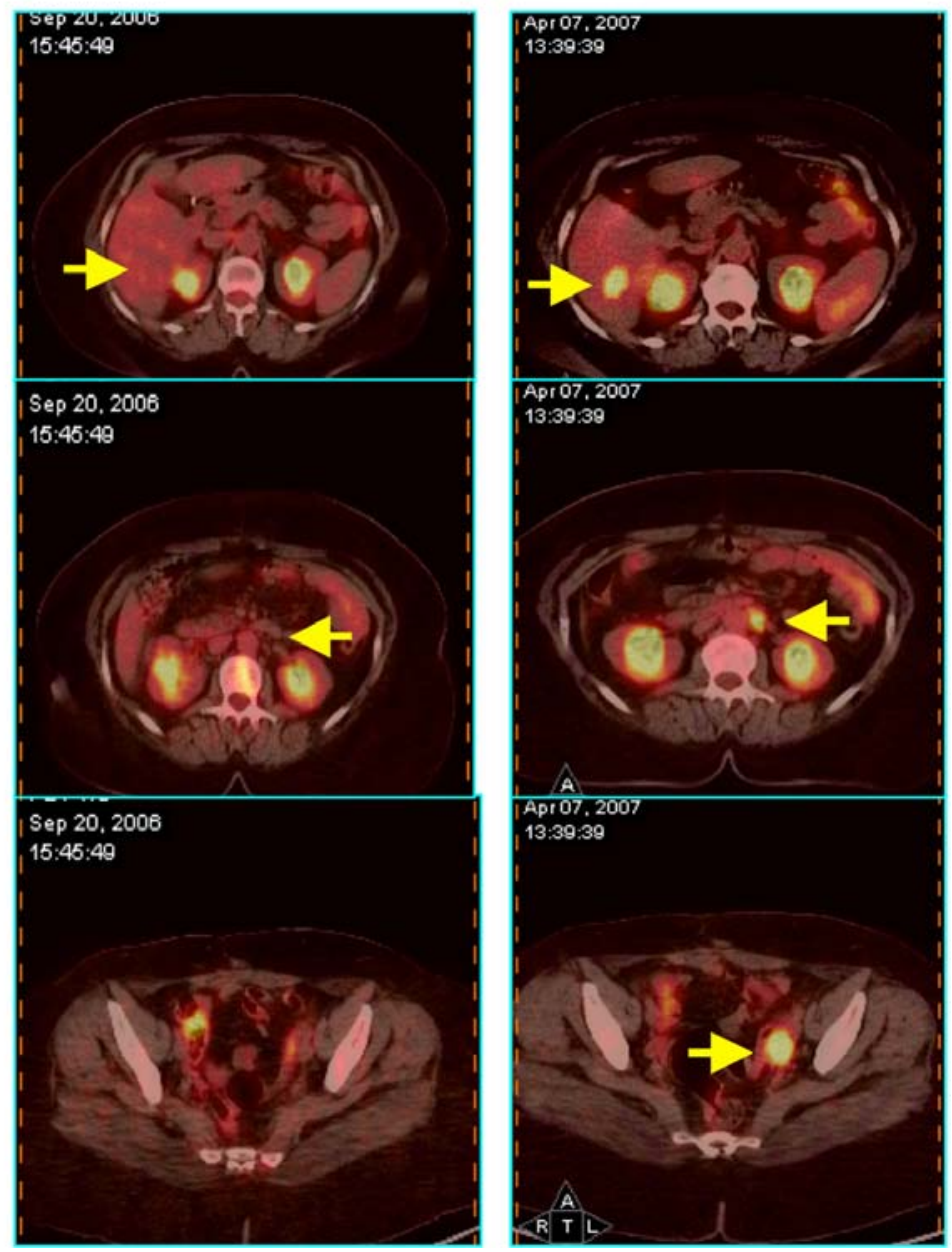

Fig. (2). Serial PET/CT studies (Pre-therapy, post therapy and follow-up) showing significant response after chemotherapy initially and recurrent disease on follow-up scan in patient with ovarian cancer. Note tumor marker correlation with PET/CT findings.

infiltration and uncontrolled formation of light chains of monoclonal immunoglobulins [55]. The diagnosis is made using laboratory parameters such as plasma protein electrophoresis and bone marrow biopsy/aspiration. M-spike on plasma protein electrophoresis is suggestive of multiple myeloma. This disease is characterized by lytic bone lesions which are present in around $80 \%$ of multiple myeloma patients [55]. Detection of bone lytic lesions is the most important factor for staging, assessing the treatment response evaluation and prognosis of multiple myeloma patients.

There are conventional and, now a days, functional imaging modalities to evaluate the status of this disease. The conventional imaging modalities have several limitations. The conventional radiography has high false negative rate and, moreover, the lytic lesions are seen on radiography only when more than $30 \%$ bone loss has occurred [56]. Whole body multidetector CT (MDCT) is better option than whole body skeletal survey but it exposes the patient to higher radiation dose. Although MRI is better than CT if the radiation safety is considered but, low dose whole body MDCT has an advantage over whole body MRI as it detects residual abnormalities in bone that are not seen by MRI [57]. $\mathrm{PET}$ and PET/CT as functional imaging modalities are more helpful in this disease for staging and treatment response evaluation. It has an advantage of scanning the whole body in less time and in a single procedure. It also detects and distinguishes the intramedullary from extramedullary lesions. Bredella et al. has reported that PET has resulted in upstaging of disease and more aggressive therapy was instituted to the patients [58]. Being a functional modality, PET accurately shows the presence of active myeloma versus monoclonal gammopathy of undetermined significance.

\section{CONCLUSION}

PET and PET/CT play an important role in evaluation treatment response during and after completion of chemotherapy in patients with various solid cancers. PET/CT studies provide both the anatomical and functional aspects of the tissue. As PET and PET/CT detect metabolic changes which happens much before structural changes in tumors, these new techniques are more sensitive in detecting early changes of therapy.

In future, changes induced by immuno-modulating drugs such as apoptosis, telomere activity, growth factor levels and many more will be studied using specific radiolabelled PET tracers using PET/CT. 


\section{REFERENCES}

[1] Kelloff, G.J.; Hoffman, J.M. Progress and promise of FDG-PET imaging for cancer patient management and oncologic drug development. Clin. Cancer. Res., 2005, 11, 2785-808.

[2] Romond, E.H.; Perez, E.A. Trastuzumab plus adjuvant chemotherapy for operable HER2-positive breast cancer. N. Engl. J. Med., 2005, 353, 1673-84.

[3] Hopkins, T.G.; Marples, M. Sunitinib in the management of gastrointestinal stromal tumours (GISTs). Eur. J. Surg. Oncol., 2008, 34, 844-50.

[4] Siddiqui, M.A.; Scott, L.J. Imatinib: a review of its use in the management of gastrointestinal stromal tumours. Drugs, 2007, 67, 805-20.

[5] Pasetto, L.M.; Bortolami, A. Recent progress in target therapy in colorectal cancer. Anticancer Res., 2006, 26, 3973-81.

[6] Moy, B.; Goss, P.E. Lapatinib: current status and future directions in breast cancer. Oncologist, 2006, 11, 1047-57.

[7] Gralow, J.R. Optimizing the treatment of metastatic breast cancer. Breast Cancer Res. Treat., 2005, 89(Suppl 1), S9-S15.

[8] Martincich, L.; Montemurro, F. Monitoring response to primary chemotherapy in breast cancer using dynamic contrast-enhanced magnetic resonance imaging. Breast Cancer Res. Treat., 2004, 83, 67-76.

[9] Wahl, R.L.; Zasadny, K. Metabolic monitoring of breast cancer chemohormonotherapy using positron emission tomography: initial evaluation. J. Clin. Oncol., 1993, 11, 2101-11.

[10] Weber, W.A. Positron emission tomography as an imaging biomarker. J. Clin. Oncol., 2006, 24, 3282-92.

[11] Smith, I.C.; Welch, A.E. Positron emission tomography using (18F)-fluorodeoxy-D-glucose to predict the pathologic response of breast cancer to primary chemotherapy. J. Clin. Oncol., 2000, 18, 1676-88.

[12] Schelling, M.; Avril, N. Positron emission tomography using [18F]fluorodeoxyglucose for monitoring primary chemotherapy in breast cancer. J. Clin. Oncol., 2000, 18, 1689-95.

[13] Rousseau, C.; Devillers, A. Monitoring of early response to neoadjuvant chemotherapy in stage II and III breast cancer by [18F] fluorodeoxyglucose positron emission tomography. J. Clin. Oncol., 2006, 24, 5366-72.

[14] Lindholm, P.; Lapela, M. Preliminary study of carbon-11 methionine PET in the evaluation of early response to therapy in advanced breast cancer. Nucl. Med. Commun., 2009, 30, 30-36.

[15] Vansteenkiste, J.; Dooms, C. Positron emission tomography in nonsmall cell lung cancer. Curr. Opin. Oncol., 2007, 19, 78-83.

[16] Wahl, R.L.; Jacene, H. From RECIST to PERCIST: Evolving considerations for PET Response Criteria in Solid Tumors. J. Nucl. Med., 2009, 50, 122S-50S.

[17] Bury, T.; Corhay, J.L. Value of FDG-PET in detecting residual or recurrent nonsmall cell lung cancer. Eur. Respir. J., 1999, 14, 1376-80.

[18] Patz, E.F.Jr.; Connolly, J. Prognostic value of thoracic FDG PET imaging after treatment for non-small cell lung cancer. AJR, 2000, 174, 769-74.

[19] Hellwig, D.; Groschel, A. Diagnostic performance and prognostic impact of FDG-PET in suspected recurrence of surgically treated non-small cell lung cancer. Eur. J. Nucl. Med. Mol. Imaging, 2006, $33,13-21$.

[20] Goudarzi, B.; Jacene, H.A. Diagnosis and differentiation of bronchioloalveolar carcinoma from adenocarcinoma with bronchioloalveolar components with metabolic and anatomic characteristics using PET/CT. J. Nucl. Med., 2008, 49, 1585-92.

[21] Nahmias, C.; Hanna, W.T. Time course of early response to chemotherapy in non-small cell lung cancer patients with 18F-FDG PET/CT. J. Nucl. Med., 2007, 48, 744-51.

[22] Jemal, A.; Siegel, R. Cancer statistics, 2008. CA. Cancer. J. Clin. 2008, 58, 71-96.

[23] Fletcher, J.W.; Djulbegovic, B. Recommendations on the use of 18F-FDG PET in oncology. J. Nucl. Med., 2008, 49, 480-508.

[24] Brucher, B.L.; Weber, W. Neoadjuvant therapy of esophageal squamous cell carcinoma: response evaluation by positron emission tomography. Ann. Surg. 2001, 233, 300-9.

[25] Flamen, P.; Van Cutsem, E. Positron emission tomography for assessment of the response to induction radiochemotherapy in locally advanced oesophageal cancer. Ann. Oncol., 2002, 13, 3618.
[26]

Swisher, S.G.; Maish, M. Utility of PET, CT, and EUS to identify pathologic responders in esophageal cancer. Ann. Thorac. Surg., 2004, 78, 1152-60.

[27] Cerfolio, R.J.; Bryant, A.S. The accuracy of endoscopic ultrasonography with fine-needle aspiration, integrated positron emission tomography with computed tomography, and computed tomography in restaging patients with esophageal cancer after neoadjuvant chemoradiotherapy. J. Thorac. Cardiovasc. Surg., 2005, 129, 1232-41.

[28] Therasse, P.; Arbuck, S.G. New guidelines to evaluate the response to treatment in solid tumors. European Organization for Research and Treatment of Cancer, National Cancer Institute of the United States, National Cancer Institute of Canada. J. Natl. Cancer Inst., 2000, 92, 205-16.

[29] Findlay, M.; Young, H. Noninvasive monitoring of tumor metabolism using fluorodeoxyglucose and positron emission tomography in colorectal cancer liver metastases: correlation with tumor response to fluorouracil. J. Clin. Oncol., 1996, 14, 700-8.

[30] Dimitrakopoulou-Strauss, A.; Strauss, L.G. Prognostic aspects of 18F-FDG PET kinetics in patients with metastatic colorectal carcinoma receiving FOLFOX chemotherapy. J. Nucl. Med., 2004, $45,1480-87$.

[31] de Geus-Oei, L.F.; van Laarhoven, H.W. Chemotherapy response evaluation with FDG PET in patients with colorectal cancer. Ann. Oncol., 2007, 19, 348-52.

[32] Langenhoff, B.S.; Oyen, W.J. Efficacy of fluorine-18deoxyglucose positron emission tomography in detecting tumor recurrence after local ablative therapy for liver metastases: a prospective study. J. Clin. Oncol., 2002, 20, 4453-8.

[33] Joosten, J.; Jager, G. Cryosurgery and radiofrequency ablation for unresectable colorectal liver metastases. Eur. J. Surg. Oncol., 2005, 31, 1152-9.

[34] Donckier, V.; Van Laethem, J.L. [F-18] fluorodeoxyglucose positron emission tomography as a tool for early recognition of incomplete tumor destruction after radiofrequency ablation for liver metastases. J. Surg. Oncol., 2003, 84, 215-23.

[35] Blokhuis, T.J.; van der Schaaf, M.C. Results of radio frequency ablation of primary and secondary liver tumors: long-term followup with computed tomography and positron emission tomography18F-deoxyfluoroglucose scanning. Scand. J. Gastroenterol., Suppl. 2004, 241, 93-7.

[36] Veit, P.; Antoch, G. Detection of residual tumor after radiofrequency ablation of liver metastasis with dualmodality PET/CT: initial results. Eur. Radiol., 2006, 16, 80-7.

[37] Ong, S.C.; Schoder, H. Clinical utility of 18F-FDG PET/CT in assessing the neck after concurrent chemoradiotherapy for locoregional advanced head and neck cancer. J. Nucl. Med., 2008, 49, 532-40.

[38] Andrade, R.S.; Heron, D.E. Posttreatment assessment of response using FDG-PET/CT for patients treated with definitive radiation therapy for head and neck cancers. Int. J. Radiat. Oncol. Biol. Phys., 2006, 65, 1315-22.

[39] Gourin, C.G.; Williams, H.T. Utility of positron emission tomography-computed tomography in identification of residual nodal disease after chemoradiation for advanced head and neck cancer. Laryngoscope, 2006, 116, 705-10.

[40] Nayak, J.V.; Walvekar, R.R. Deferring planned neck dissection following chemoradiation for stage IV head and neck cancer: the utility of PETCT. Laryngoscope, 2007, 117, 2129-34.

[41] Kumar, R.; Xiu, Y. 18F-FDG PET for evaluation of the treatment response in patients with gastrointestinal tract lymphomas. J. Nucl. Med., 2004, 45, 1796-803.

[42] Cachin, F.; Kelly, A. Evaluation of the therapeutic response: role of isotopic imaging. Bull. Cancer, 2006, 93, 1191-9.

[43] Kasamon, Y.L.; Jones, R.J. Integrating PET and PET/CT into the risk-adapted therapy of lymphoma. Leuk. Lymphoma., 2007, 48, 270-82.

[44] Specht, L. 2-[18F] fluoro-2-deoxyglucose positron-emission tomography in staging, response evaluation, and treatment planning of lymphomas. Semin. Radiat. Oncol., 2007, 17, 190-7.

[45] van der Hiel, B.; Pauwels, E.K. Positron emission tomography with 2-[18F]-fluoro-2-deoxy-D-glucose in oncology. Part IIIa: Therapy response monitoring in breast cancer, lymphoma and gliomas. $J$. Cancer. Res. Clin. Oncol., 2001, 127, 269-77.

[46] Zhao, J.; Qiao, W. Therapeutic evaluation and prognostic value of interim hybrid PET/CT with (18)F-FDG after three to four cycles 
of chemotherapy in non-Hodgkin's lymphoma. Hematology 2007, 12, 423-30.

[47] Grigsby, P.W.; Siegel, B.A. Posttherapy [18F] fluorodeoxyglucose positron emission tomography in carcinoma of the cervix: response and outcome. J. Clin. Oncol., 2004, 22, 2167-71.

[48] Schwarz, J.K.; Siegel, B.A. Association of posttherapy positron emission tomography with tumor response and survival in cervical carcinoma. JAMA, 2007, 298, 2289-95.

[49] Kumar, R.; Dadparvar, S. ${ }^{18}$ F-fluoro-2-deoxy-d-glucose-positron emission tomography (PET)/PET-computed tomography in carcinoma of the cervix. Cancer, 2007, 30, 1650-3.

[50] Lin, L.L.; Yang, Z. FDG-PET imaging for the assessment of physiologic volume response during radiotherapy in cervix cancer. Int. J. Radiat. Oncol. Biol. Phys., 2006, 65, 177-81.

[51] Schwarz, J.K.; Lin, L.L. 18-F-fluorodeoxyglucosepositron emission tomography evaluation of early metabolic response during radiation therapy for cervical cancer. Int. J. Radiat. Oncol. Biol. Phys., 2008, 72, 1502-7.

[52] Avril, N.; Sassen, S. Prediction of response to neoadjuvant chemotherapy by sequential F-18-fluorodeoxyglucose positron emission tomography in patients with advanced-stage ovarian cancer. J. Clin. Oncol., 2005, 23, 7445-53.
[53] Nishiyama, Y.; Yamamoto, Y. Monitoring the neoadjuvant therapy response in gynecological cancer patients using FDG PET. Eur. $J$. Nucl. Med. Mol. Imaging, 2008, 35, 287-95.

[54] Landis, SH.; Murray, T. Cancer statistics, 1998. CA. Cancer J. Clin., 1998, 48, 6-29.

[55] Nanni, C.; Zamagni, E. Role of 18F-FDG PET/CT in the assessment of bone involvement in newly diagnosed multiple myeloma: preliminary results. Eur. J. Nucl. Med. Mol. Imaging, 2006, 33, 525-31.

[56] Baur-Melnyk, A.; Reiser, M. Staging of multiple myeloma with MRI: comparison to MSCT and conventional radiography. Radiologe, 2004, 44, 874-81.

[57] Gleeson, TG.; Moriarty, J. Accuracy of whole-body low-dose multidetector CT (WBLDCT) versus skeletal survey in the detection of myelomatous lesions, and correlation of disease distribution with whole-body MRI (WBMRI). Skeletal Radiol., 2009, 38, 225-36.

[58] Bredella, MA.; Steinbach, L. Value of FDG PET in the assessment of patients with multiple myeloma. Am. J. Roentgenol., 2005, 184, 1199-204.

(C) Kumar et al.; Licensee Bentham Open.

This is an open access article licensed under the terms of the Creative Commons Attribution Non-Commercial License (http://creativecommons.org/licenses/by-nc/3.0/) which permits unrestricted, non-commercial use, distribution and reproduction in any medium, provided the work is properly cited. 